\title{
THE INFLUENCE OF SOURCE TERMS ON STABILITY AND CONSERVATION IN 1D HYPERBOLIC SYSTEM OF EQUATIONS: APLICATION TO FIXED AND MOVABLE BEDS
}

\author{
J. Murillo ${ }^{\dagger}$, P. Garcia-Navarro ${ }^{\dagger}$ and J. Burguete \\ ${ }^{\dagger}$ Fluid Mechanics, CPS, University of Zaragoza, Spain \\ ${ }^{\ddagger}$ E.E.A.D., C.S.I.C., Zaragoza, Spain
}

KEYWORDS: Source terms, stability condition, wetting/drying, MUSCL-Hancock, unstructured grids, two-dimensional hydraulic model.

\begin{abstract}
The non conservative terms that appear in systems of conservation laws poses an extra difficulty when managing practical cases. The influence of this terms in the stability region for finite volume schemes is analyzed under the hypothesis of a well balanced scheme, based in the $C$-Property. It is demonstrated that in each particular situation the stability region is amplified or reduced, and how in some special cases depends on the initial conditions. The time step requirements for stability are defined at the cell edges, related with the traditional Courant-Friedrichs-Lewy (CFL) condition. It is applied to the shallow water equations, to the shallow water equations with solute transport and to sediment transport problems.
\end{abstract}

\section{INTRODUCTION}

In the basis of a one-dimensional mathematical model for debris flow, Brufau et al., 2000 presented a Godunov-type finite volume scheme, based on Roe's numerical approach (Roe, 1986). Hudson and Sweby, (Hudson and Sweby, 2003) investigated the equations governing bed-load sediment transport for steady and an unsteady approaches using a flux-limited version of Roe's scheme and and extended it to the two-dimensional case (Hudson and Sweby, 2005) requiring an exact balance between the flux gradient and the source term, following the idea of exact conservation ( $\mathcal{C}$-property) introduced by Bermudez and Vazquez (Bermudez and Vazquez, 1994). Crnjaric-Zic et al, 2004 extend another type of Godunov-type schemes, ENO and WENO, to the one dimensional sediment transport equations, using the mathematical model presented by Hudson, (Hudson, 2001) requiring the $\mathcal{C}$-property. In their work the trust term was included in the Jacobian matrix of the flux assuming by means of a local linearization, remaining the system hyperbolic. Also the $\mathcal{C}$-property was required in the case of quiescent flow. Rosatti and Fraccarollo, 2006 presented a mathematical model for high sediment transport that was solved by means of a Godunov-type scheme requiring $\mathcal{C}$-property leading to accurate results.

In Murillo et al., 2006 the stability region for systems of conservation laws was analyzed, making emphasis in the numerical conservation and in the preservation of the positivity property of the solution when necessary in the presence of source terms. The numerical approach was based in the approximate Roe's solver, and applied to the shallow water equations, where the source term treatment satisfied $\mathcal{C}$-property including both bed and friction terms, leading to an exact balance among fluxes and non conservative terms, not only in the case of quiescence flow over discontinuous bed topography, also in the case of steady fluid in motion. 
This is a conservation law expressing that a function $u$ varies and is transported according to both the distribution of a flux function $f$ and to a source term $s$, in the form:

$$
\partial_{t} u+\partial_{x} f=s(u, x)
$$

At this point, the source term is assumed to follow $s=\partial_{x} \tau$, and the advection, or transport, velocity $\lambda$ is:

$$
\lambda=\frac{d f}{d u}
$$

To introduce the upwind finite volume scheme, (1) is integrated in a volume $\Omega$ :

$$
\frac{\partial}{\partial t} \int_{\Omega} u(x, y) d \Omega+\int_{\Omega} \partial_{x}(f-\tau) d \Omega=0
$$

where $d \Omega$ denotes the contour line. If Gauss's theorem is applied to the second integral in (3):

$$
\frac{\partial}{\partial t} \int_{\Omega} u d \Omega+\int_{\partial \Omega}(f-\tau) \mathrm{d} l=0
$$

In the one-dimensional case plane, the volumes are actually lines, and considering a regular mesh, $\Omega=\Delta x$ and $\mathrm{d} l$ become unity. Now, a cell-centred finite volume method can be formulated where all the dependent variables of the system are represented as piecewise constants (first order). Therefore quantities $u, f$ and $\tau$ are uniform per cell of length $\Delta x$. In particular, the first integral in (4) can be approximated by:

$$
\frac{\partial}{\partial t} \int_{\Omega} u d \Omega=\frac{\partial u_{i}}{\partial t} \Delta x \cong \frac{u_{i}^{n+1}-u_{i}^{n}}{\Delta t} \Delta x
$$

In the second integral of (16) the flux $f$ is:

$$
\int_{\partial \Omega} f=f_{i+1}-f_{i-1}=\delta f_{i+1 / 2}+\delta f_{i-1 / 2}
$$

with $\delta f_{i+1 / 2}=f\left(u_{i+1}\right)-f\left(u_{i}\right)=f_{i+1}-f_{i}$.

Using (2), the linearized advection velocity $\tilde{\lambda}_{i+1 / 2}$ can be defined (Roe, 1986) as:

$$
\widetilde{\lambda}_{i+1 / 2}=\frac{\left(f_{i+1}-f_{i}\right)}{\left(u_{i+1}-u_{i}\right)}
$$

Following the upwind philosophy, which discriminates the sense of propagation according to the sign of the advection velocity, the flux difference is split (Roe, 1986) as a sum of waves travelling in and out of a given cell:

$$
\delta f_{i+1 / 2}=\delta f_{i+1 / 2}^{-}+\delta f_{i+1 / 2}^{+}=\tilde{\lambda}_{i+1 / 2}^{-} \delta u_{i+1 / 2}+\tilde{\lambda}_{i+1 / 2}^{+} \delta u_{i+1 / 2}
$$

with $\widetilde{\lambda}^{ \pm}=(\widetilde{\lambda} \pm|\widetilde{\lambda}|) / 2 \widetilde{\lambda}_{i+1 / 2}=\widetilde{\lambda}_{i+1 / 2}^{-}+\widetilde{\lambda}_{i+1 / 2}^{+} \cdot 0$

In the case of the source term $\tau$ :

$$
\int_{\Delta x} \tau=\tau_{i+1}-\tau_{i}=\delta \tau_{i+1 / 2}+\delta \tau_{i-1 / 2}
$$

The edge source term $\delta \tau_{i+1 / 2}$ is also split into in-going and out-going contributions

$$
\delta \tau_{i+1 / 2}=(\delta \tau)_{i+1 / 2}^{-}+(\delta \tau)_{i+1 / 2}^{+}
$$

where

$$
(\delta \tau)_{i+1 / 2}^{ \pm}=\frac{1}{2}\left(1 \pm \operatorname{sgn}\left(\widetilde{\lambda}_{i+1 / 2}^{ \pm}\right)\right)(\delta \tau)_{i+1 / 2}
$$

The updating scheme for cell $i$ includes only the in-going contribution of flux and source term to that cell. 


$$
u_{i}^{n+1}=u_{i}^{n}-\left[\delta(f-\tau)_{i-/ 2}^{+}+\delta(f-\tau)_{i+/ 2}^{-}\right] \frac{\Delta t}{\Delta x}
$$

Equation (14) is the later so called unified discretization. This can also be expressed in a compact form as:

$$
u_{i}^{n+1}=u_{i}^{n}-\left[v_{i-/ 2}^{+} \delta u_{i-/ 2}+v_{i+/ 2}^{-} \delta u_{i+/ 2}\right], \quad v_{i+1 / 2}^{ \pm}=\lambda_{i \mp 1 / 2}^{*}, \frac{\Delta t}{\Delta x}
$$

with

$$
\lambda_{i \mp 1 / 2}^{*, \pm}=\lambda_{i \mp 1 / 2}^{ \pm} \theta_{i+1 / 2}^{ \pm} \quad \theta_{i+1 / 2}^{ \pm}=1-\left(\frac{\delta \tau}{\tilde{\lambda} \delta u}\right)_{i \mp 1 / 2}^{ \pm}=1-\left(\frac{\delta \tau^{-}}{\delta f^{-}}\right)_{i \mp 1 / 2}^{ \pm}
$$

Note that $v_{i \mp 1 / 2}^{ \pm}$is a dimensionless quantity that plays the role of a local CFL number generalized to cases involving source terms. The coefficient $\theta_{i \mp 1 / 2}^{ \pm}$expresses the discrete ratio of source term to flux differences. They will be key parameters in our discussion of stability conditions.

\section{Influence of $\theta_{i+1 / 2}^{ \pm}$on the stability condition}

In the homogeneous case $\theta_{i+1 / 2}^{ \pm}=1$. Then, numerical stability for scheme (14) is ensured if

$$
0 \leq v_{i-1 / 2}^{+} \leq 1, \quad-1 \leq v_{i+1 / 2}^{-} \leq 0
$$

At the same time, the following condition on the monotonicity of the solution holds

$$
u^{\min } \leq u_{i}^{n+1} \leq u^{\max }
$$

where $u^{\max }=\max \left\{u_{i}^{n}, u_{i+1}^{n}, u_{i-1}^{n}\right\}$ and $u^{\min }=\min \left\{u_{i}^{n}, u_{i+1}^{n}, u_{i-1}^{n}\right\}$.

The aim of the following analysis is to find the criterion that preserves (16) in presence of source terms. For that reason, first it is necessary to enforce (16) by requiring

this is possible when

$$
\tilde{\lambda}_{i-1 / 2}^{+, *} \geq 0, \quad \tilde{\lambda}_{i+1 / 2}^{-* *} \leq 0
$$

$$
\theta_{i \mp 1 / 2}^{ \pm} \geq 0
$$

Given a grid mesh and initial data values, (16) is a limit on the value of the time step to meet the stability criterion. For the sake of simplicity, assume that all the $u_{i+1}$ and $u_{i-1}$ values are equal but $u_{i}>u_{i+1}$. The size of the allowable incoming contributions are bounded by

$$
\left|v_{i-/ 2}^{+} \delta u_{i-/ 2}+v_{i+/ 2}^{-} \delta u_{i+/ 2}\right| \leq \lambda_{\max }^{*} \delta u_{o} \frac{\Delta t}{\Delta x}
$$

where $\lambda_{\max }^{*}=\max \left\{\left|\lambda_{i-1+2}^{*++}\right|,\left|\lambda_{i+1+2}^{*--}\right|\right\}$ and $\delta u_{o}=u_{i}-u_{i+1}$. As the updating flux must be limited by the quantity that ensures that the final state is included between the initial values $\delta u_{o}$, the following is also true:

$$
\lambda_{\max }^{*} \delta u_{o} \frac{\Delta t}{\Delta x} \leq \delta u_{o}
$$

Under these conditions maximum allowable time step is formulated in terms of the edge-time steps as follows:

$$
\begin{gathered}
\Delta t=C F L \Delta t_{\max }, \quad C F L \leq 1 \\
\Delta t_{\max }=\min \left\{\Delta t_{i+1 / 2}\right\}_{k=1, N} \quad \Delta t_{i+1 / 2}=\frac{\Delta x}{\left|\lambda_{i+1 / 2}^{*}\right|}, \quad \theta_{i+1 / 2} \geq 0
\end{gathered}
$$

If $\theta_{i+1 / 2}$ is set equal to one in (21), the basic CFL stability condition for the homogeneous case is automatically recovered. Otherwise, (21) states a more general rule. At this point it is 
worth remarking the relevance of the source term discretization when analysing the stability region defined by (23). If a unified formulation has been used so that in equilibrium

$$
(\delta(f-\tau))_{i+1 / 2}^{-}=0 \quad(\delta(f-\tau))_{i-1 / 2}^{+}=0
$$

This means that $\theta_{i \mp 1 / 2}^{ \pm}=0$ and therefore $\lambda_{i \mp 1 / 2}^{*, \pm}=0$. The numerical scheme becomes unconditionally stable at steady state, and $\mathcal{C}$-Property is achieved.

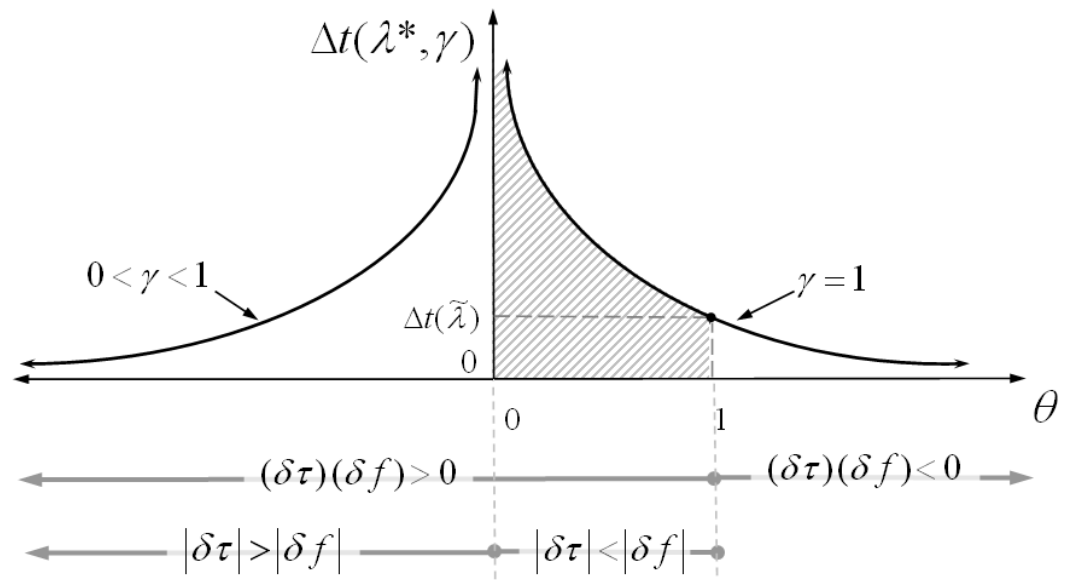

Figure 1. Stability region. First order scheme in presence of source terms

Figure 1 represents the stability region of the scheme as a function of $\theta$. The point $\theta=1$ on the curve corresponds to the homogeneous case (no source term) and $\Delta t\left(\lambda_{i+1 / 2}^{*}\right)$ is the maximum time step compatible with stability in this case or CFL condition (Courant et al. 1952). The rest of the curve corresponds to cases with source terms. In Figure 3 the sign relations among fluxes and source terms are displayed. The dashed zone $(0 \leq \theta \leq 1)$ is the set of situations in which it could be possible to use larger time steps than the limit of the homogeneous stability: both fluxes and source terms have the same sign so the net contributions are reduced. In the case $\theta>1$, stability requires a reduction of the time step size over that dictated by the CFL condition, as fluxes and source terms have an opposite sign, so the net contribution increases.

On the other hand it is possible to find situations where $\theta_{i+1 / 2}<0$. In that case the source term contribution dominates over the flux difference, $\left|\delta \tau^{-}\right|_{i+1 / 2}>\left|\delta f^{-}\right|_{i+1 / 2}$ and a different line of reasoning must be followed. Depending on the requirements over the solution different strategies can be tackled. If preservation of the sign of the variable is the main objective,

$$
u_{i}^{n+1} \leq 0 \quad \text { when } u_{i}^{n}, u_{i \pm 1}^{n} \leq 0 \quad, \quad u_{i}^{n+1} \geq 0 \quad \text { when } \quad u_{i}^{n}, u_{i \pm 1}^{n} \geq 0
$$

the contributions between cells are also limited by the initial values stored in the cells so that the stability condition is determined also by the initial condition:

$$
\Delta t_{i+1 / 2}=\gamma \frac{\Delta x}{\left|\lambda_{i+1 / 2}^{*}\right|}, \quad \gamma=\frac{\min \left\{\left|u_{i}\right|,\left|u_{i+1}\right|,\left|\delta u_{i+1 / 2}\right|\right\}}{\left|\delta u_{i+1 / 2}\right|}, \quad \theta_{i+1 / 2}^{ \pm}<0
$$

If $u$ is a gradually varied function, the coefficient $\gamma$ is 1 and the time step limit in (24) reduces to (21). Otherwise, $0 \leq \gamma<1$ and an actual reduction in the time step is required. In the special case $\gamma=0$ the local time step $\Delta t_{i+1 / 2}$ would be zero according to (24). This is absurd and must be interpreted as condition of no information crossing that cell edge. In 
practice, a threshold value equal to the machine accuracy is defined for the minimum value of $\gamma$ before imposing the condition that no information crosses the edge.

\section{SYSTEMS OF CONSERVATION LAWS WITH SOURCE TERMS}

The numerical methods are extended in this chapter to solve hyperbolic non-linear systems of equations with source terms, of the form:

$$
\partial_{t} \mathbf{U}+\partial_{x} \mathbf{F}(\mathbf{U})=\mathbf{S}(\mathbf{U}, x)
$$

It will be first assumed that the source term $\mathbf{S}$ can be expressed as:

$$
\mathbf{S}(\mathbf{U})=\partial_{x} \mathbf{T}
$$

so (26) becomes:

$$
\partial_{t} \mathbf{U}+\vec{\nabla}(\mathbf{F}(\mathbf{U})-\mathbf{T}(\mathbf{U}))=0
$$

The mathematical properties of the hyperbolic system of equations include the existence of a Jacobian matrix, $\mathbf{J}$, of the flux $\mathbf{F}$ defined as

$$
\mathbf{J}=\frac{\partial \mathbf{F}}{\partial \mathbf{U}}
$$

From its eigenvectors, two matrices $\mathbf{P}$ and $\mathbf{P}^{-1}$ can be constructed with the property that they diagonalize the Jacobian $\mathbf{J}$,

$$
\mathbf{J}=\mathbf{P} \Lambda \mathbf{P}^{-1}
$$

where $\boldsymbol{\Lambda}$ is a diagonal matrix with eigenvalues in the main diagonal.

The equivalent to (3) for the system is

$$
\frac{\partial}{\partial t} \int_{\Omega} \mathbf{U} d \Omega+\int_{\Omega} \nabla(\mathbf{F}-\mathbf{T}) d \Omega=0
$$

If Gauss's theorem is applied to the second integral in (19):

$$
\frac{\partial}{\partial t} \int_{\Omega} \mathbf{U} d \Omega+\int_{\partial \Omega}(\mathbf{F}-\mathbf{T}) \mathrm{d} l=0
$$

Now, a cell-centred finite volume method can be formulated where all the dependent variables of the system are represented as piecewise constants (first order). Therefore the vector quantities $\mathbf{U}, \mathbf{F}$ and $\mathbf{T}$ are uniform per cell of length $\Delta x$. In particular, the first integral in (20) can be approximated by:

$$
\frac{\partial}{\partial t} \int_{\Omega} \mathbf{U}(x, y) d \Omega=\frac{\partial \mathbf{U}_{i}}{\partial t} \Delta x \cong \frac{\mathbf{U}_{i}^{n+1}-\mathbf{U}_{i}^{n}}{\Delta t} \Delta x
$$

In the second integral of (20) the flux $\mathbf{F}$ becomes:

$$
\int_{\partial \Omega} \mathbf{F}=\mathbf{F}_{i+1}-\mathbf{F}_{i-1}=\delta \mathbf{F}_{i+1 / 2}+\delta \mathbf{F}_{i-1 / 2}
$$

with $\delta \mathbf{F}_{i+1 / 2}=\mathbf{F}\left(\mathbf{U}_{i+1}\right)-\mathbf{F}\left(\mathbf{U}_{i}\right)=\mathbf{F}_{i+1}-\mathbf{F}_{i}$.

Due to the non-linear character of the flux $\mathbf{F}$, the definition of an approximated flux Jacobian, $\widetilde{\mathbf{J}}_{i+1 / 2}$ (Roe, 1986) allows for a local linearization and is exploited here. Roe suggested that the following conditions should be imposed on the approximate matrix $\widetilde{\mathbf{J}}_{i+1 / 2}$ :

i) $\widetilde{\mathbf{J}}_{i+1 / 2}=\widetilde{\mathbf{J}}_{i+1 / 2}\left(\mathbf{U}_{i}, \mathbf{U}_{i+1}\right)$

ii) $\mathbf{F}_{i+1}-\mathbf{F}_{i}=\widetilde{\mathbf{J}}_{i+1 / 2}\left(\mathbf{U}_{i+1} \cdot-\mathbf{U}_{i}\right)$

iii) $\widetilde{\mathbf{J}}_{i+1 / 2}$ has real eigenvalues and a set of eigenvectors

iv) $\widetilde{\mathbf{J}}_{i+1 / 2}=\widetilde{\mathbf{J}}_{i+1 / 2}\left(\mathbf{U}_{i+1}\right)=\widetilde{\mathbf{J}}_{i+1 / 2}\left(\mathbf{U}_{i}\right)$ if $\mathbf{U}_{i+1}=\mathbf{U}_{i}$ 
Matrices $\widetilde{\mathbf{P}}^{-1}$ and $\widetilde{\mathbf{P}}$ can be built so that they diagonalize the approximate Jacobian matrix $\widetilde{\mathbf{J}}_{i+1 / 2}$

$$
\widetilde{\mathbf{J}}_{i+1 / 2}=\left(\widetilde{\mathbf{P}} \widetilde{\mathbf{\Lambda}} \widetilde{\mathbf{P}}^{-1}\right)_{i+1 / 2}, \quad \widetilde{\mathbf{P}}_{i+1 / 2}=\left[\widetilde{\mathbf{e}}^{1}, \ldots ., \widetilde{\mathbf{e}}^{N \lambda}\right]_{i+1 / 2}
$$

and $\widetilde{\Lambda}$ is the diagonal eigenvalues matrix. From the approximate Jacobian (Roe, 1986)

$$
\widetilde{\mathbf{J}}_{i+1 / 2} \widetilde{\mathbf{e}}_{i+1 / 2}^{m}=(\widetilde{\lambda} \widetilde{\mathbf{e}})_{i+1 / 2} \quad m=1, \ldots, N \lambda
$$

where $N \lambda$ is the number of eigenvalues, $\tilde{\lambda}^{m}$. Following a flux difference procedure, the difference in vector $\mathbf{U}$ across the grid edge is projected onto the matrix eigenvectors basis

$$
\delta \mathbf{U}_{i+1 / 2}=\sum_{m=1}^{N \lambda}(\alpha \widetilde{\mathbf{e}})_{i+1 / 2}^{m}
$$

Now the contributions in (33) are written as:

$$
\widetilde{\mathbf{J}}_{i-1 / 2} \delta \mathbf{U}_{i-1 / 2}+\widetilde{\mathbf{J}}_{i+1 / 2} \delta \mathbf{U}_{i+1 / 2}=\sum_{m=1}^{N_{\lambda}}\left(\widetilde{\lambda}^{m} \alpha^{m} \widetilde{\mathbf{e}}^{m}\right)_{i+1 / 2}+\sum_{m=1}^{N_{\lambda}}\left(\widetilde{\lambda}^{m} \alpha^{m} \widetilde{\mathbf{e}}^{m}\right)_{i+1 / 2}
$$

Since all the variables defined at the cell are uniform, the term $\mathbf{T}$ in the second integral in (31), is approximated by

$$
\int_{\partial \Omega} \mathbf{T n} \mathrm{d} l=\mathbf{T}_{i+1}-\mathbf{T}_{i-1}=\delta \mathbf{T}_{i+1 / 2}+\delta \mathbf{T}_{i-1 / 2}
$$

The normal source difference $\delta \mathbf{T}_{i+1 / 2}$ can also be expressed in function of the eigenvalues and eigenvectors of $\widetilde{\mathbf{J}}_{i+1 / 2}$, using the approximate matrix $\widetilde{\mathbf{P}}_{i+1 / 2}$ in order to reach a unified formulation:

$$
\delta \mathbf{T}_{i+1 / 2}=\sum_{m=1}^{N_{\lambda}}\left(\beta \widetilde{\mathbf{e}}^{m}\right)_{i+1 / 2}^{m}
$$

where the different $\beta^{m}$ coefficients are computed as

$$
\boldsymbol{\beta}_{i+1 / 2}=\widetilde{\mathbf{P}}_{i+1 / 2}^{-1} \delta \mathbf{T}_{i+1 / 2}
$$

with $\boldsymbol{\beta}_{i+1 / 2}=\left[\beta^{1}, \ldots ., \beta^{m}\right]_{i+1 / 2}^{T}$.

In order to discriminate the sense of advection linked to the sign of the different eigenvalues, two matrices $\widetilde{\Lambda}^{ \pm}$are defined:

$$
\widetilde{\boldsymbol{\Lambda}}^{ \pm}=(\widetilde{\boldsymbol{\Lambda}} \pm|\widetilde{\boldsymbol{\Lambda}}|) / 2
$$

The flux difference across each edge $i+1 / 2$ is split into contributions directed to cell $i+1$ (positive waves) and contributions directed to cell $i$ (negative waves):

$$
\widetilde{\mathbf{J}}_{i+1 / 2} \delta \mathbf{U}_{i+1 / 2}=\left(\widetilde{\mathbf{P}} \widetilde{\boldsymbol{\Lambda}}^{-} \widetilde{\mathbf{P}}^{-1}\right)_{i+1 / 2} \delta \mathbf{U}_{i+1 / 2}+\left(\widetilde{\mathbf{P}} \widetilde{\boldsymbol{\Lambda}}^{+} \widetilde{\mathbf{P}}^{-1}\right)_{i+1 / 2} \delta \mathbf{U}_{i+1 / 2}
$$

For the updating algorithm, as defined for a given cell $i$, only the contributions directed to cell $i$ generated at the edges $i+1 / 2$ and $i-1 / 2$ are of interest. The contour integral of the numerical normal flux is equivalent to the sum of the in-going waves:

$$
\widetilde{\mathbf{J}}_{i-1 / 2} \delta \mathbf{U}_{i-1 / 2}+\widetilde{\mathbf{J}}_{i+1 / 2} \delta \mathbf{U}_{i+1 / 2} \cong \sum_{m=1}^{N_{\lambda}}\left(\widetilde{\lambda}^{+m} \alpha^{m} \widetilde{\mathbf{e}}^{m}\right)_{i-1 / 2}+\sum_{m=1}^{N_{\lambda}}\left(\widetilde{\lambda}^{-m} \alpha^{m} \widetilde{\mathbf{e}}^{m}\right)_{i+1 / 2}
$$

where $\lambda^{-}=\frac{1}{2}(\lambda-|\lambda|)$.

In order to enforce an exact equilibrium in steady state cases the normal source difference $\delta \mathbf{T}_{i+1 / 2}$ is be split in two kinds of waves:

$$
\delta \mathbf{T}_{i+1 / 2}=(\delta \mathbf{T} \mathbf{n})_{i+1 / 2}^{+}+(\delta \mathbf{T} \mathbf{n})_{i+1 / 2}^{-}
$$

with the same philosophy as before and where 


$$
\delta \mathbf{T}_{i+1 / 2}^{ \pm}=\sum_{m=1}^{N_{\lambda}}(\beta \widetilde{\mathbf{e}})_{i+1 / 2}^{ \pm m}
$$

with $\beta^{ \pm m}=\frac{1}{2}\left(1 \pm \operatorname{sgn}\left(\widetilde{\lambda}^{m}\right)\right) \beta^{m}$.

The first order upwind scheme gets the form:

$$
\mathbf{U}_{i}^{n+1}=\mathbf{U}_{i}^{n}-\frac{\Delta t}{\Delta x}\left(\sum_{m=1}^{N_{\lambda}}\left(\left(\tilde{\lambda}^{+} \alpha-\beta^{+}\right) \widetilde{\mathbf{e}}\right)_{i-1 / 2}^{m}+\sum_{m=1}^{N_{\lambda}}\left(\left(\tilde{\lambda}^{-} \alpha-\beta^{-}\right) \widetilde{\mathbf{e}}\right)_{i+1 / 2}^{m}\right)
$$

This is a compact form with the focus on the waves generated at the cell edges, made of both the normal flux difference and the normal source term, and governed by the sign of the eigenvalues of the normal flux Jacobian.

As was done in the scalar case, the numerical scheme in (47) can be rewritten as:

$$
\mathbf{U}_{i}^{n+1}=\mathbf{U}_{i}^{n}-\left[\sum_{m=1}^{N \lambda} v_{i-/ 2}^{m,+} \delta \mathbf{U}_{i-/ 2}+\sum_{m=1}^{N \lambda} v_{i+/ 2}^{m,-} \delta \mathbf{U}_{i+/ 2}\right], \quad v_{i \mp 1 / 2}^{m, \pm}=\lambda_{i \mp 1 / 2}^{m^{*}, \pm} \frac{\Delta t}{\Delta x}
$$

with

$$
\lambda_{i \mp 1 / 2}^{m^{*}, \pm}=\lambda_{i \mp 1 / 2}^{m \pm} \theta_{i \mp 1 / 2}^{m \pm} \quad \theta_{i \mp 1 / 2}^{m \pm}=1-\left(\frac{\beta^{-}}{\alpha \tilde{\lambda}^{-}}\right)_{i \mp 1 / 2}^{ \pm}
$$

where the ratio $\beta /(\alpha \tilde{\lambda})$ expresses the influence of the source terms over that of the flux differences.

In absence of source terms, the numerical scheme (47) is stable provided that

$$
-1 \leq v_{i+1 / 2}^{m,-} \leq 0 \quad 0 \leq v_{i-1 / 2}^{m .+} \leq 1 \quad m=1, \ldots, N \lambda
$$

and the following condition over the conserved variables applies:

$$
U_{s}^{\min } \leq U_{s, i}^{n+1} \leq U_{s}^{\max } \quad \mathbf{U}=\left(U_{1}, \ldots, U_{s}, \ldots, U_{n c}\right)^{T}
$$

where $U_{S}^{\max }=\max \left\{U_{S, i}^{n}, U_{S, i+1}^{n}, U_{S, i-1}^{n}\right\}$ and $U_{S}^{\min }=\min \left\{U_{S, i}^{n}, U_{S, i+1}^{n}, U_{S, i-1}^{n}\right\}$.

Monotonicity in the conserved variables in presence of source terms requires that for all $m$

which means that

$$
\widetilde{\lambda}_{i+1 / 2}^{m-*} \leq 0 \quad \widetilde{\lambda}_{i-1 / 2}^{m+, *} \geq 0 \quad m=1, \ldots, N \lambda
$$

$$
\theta_{i \mp 1 / 2}^{m, \pm} \geq 0 \quad m=1, \ldots, N \lambda
$$

Under these assumptions the numerical stability when $\theta_{i \mp 1 / 2}^{m, \pm} \geq 0$ for all $m$, is provided by the intersection of the stability regions defined for each celerity $\tilde{\lambda}_{i+1 / 2}^{m, *}$

$$
\begin{gathered}
\Delta t=C F L \Delta t_{\max }, \quad C F L \leq 1 \\
\Delta t_{\max }=\min \left\{\Delta t_{i+1 / 2}\right\}_{k=0, N} \quad \Delta t_{i+1 / 2}=\frac{\Delta x}{\max _{m}\left\{\left|\lambda_{i+1 / 2}^{m, *}\right|\right\}}, \quad \theta_{i+1 / 2}^{m, \pm} \geq 0 \quad m=1, \ldots, N \lambda
\end{gathered}
$$

In the particular case of $\theta_{i+1 / 2}^{m, \pm}=1$ for all $m$, (54) expresses the stability condition without source terms (CFL condition).

Equilibrium in steady state cases is ensured if the discretization of the source term has been constructed enforcing

$$
(\tilde{\lambda} \alpha-\beta)_{i \mp 1 / 2}^{m \pm}=0 \quad m=1, \ldots, N \lambda
$$

which is equivalent to $\lambda_{i \mp 1 / 2}^{m \pm}=0$, leading to an unconditionally stable scheme in this particular case.

Equation (47) can also be expressed 


$$
\mathbf{U}_{i}^{n+1}=\mathbf{U}_{i}^{n}-\left[\sum_{m=1}^{N \lambda} \tilde{\lambda}_{i-/ 2}^{m,+} \delta \mathbf{D}_{i-/ 2}+\sum_{m=1}^{N \lambda} \tilde{\lambda}_{i+/ 2}^{m,-} \delta \mathbf{D}_{i+/ 2}\right] \frac{\Delta t}{\Delta x}, \quad \delta \mathbf{D}_{i \pm 1 / 2}^{m}=\theta_{i \pm 1 / 2}^{m} \delta \mathbf{U}_{i \pm 1 / 2}^{m}
$$

The stability region for (56) with source terms is enlarged if $0 \leq \theta_{i \pm 1 / 2}^{m} \leq 1$ for all $m$, as in this particular case $\left|\delta \mathbf{D}_{k}^{m}\right| \leq\left|\delta \mathbf{U}_{k}^{m}\right|$. Equilibrium is achieved when all $\delta \mathbf{D}_{k}^{m}=0$, condition that can be automatically derived from (55).

On the other hand, as seen in the scalar case, when $\theta_{i \pm 1 / 2}^{m}<0$ the source terms dominate over the flux differences. If (51) is still desired, the definition of $\gamma$ has to be based on the specific necessities of the physical problem. A direct extension of (24) is not possible, as it is not feasible to define a $\gamma_{i+1 / 2}^{m}$ coefficient if $\theta_{i \pm 1 / 2}^{m}<0$ for all $m$, since there is not a correspondence between the $m$-waves and the $s$-variables. In the special case trying to preserve the sign over the solution in the $s$ component, expressed as

$$
U_{s, i}^{n+1} \leq 0 \text { with } U_{s, i}^{n}, U_{s, i \pm 1}^{n} \leq 0 \quad \text { or } \quad U_{s, i}^{n+1} \geq 0 \text { with } U_{s, i}^{n}, U_{s, i \pm 1}^{n} \geq 0
$$

the time step in the stability region must be computed following

$$
\Delta t_{i+1 / 2}=\gamma \frac{\Delta x}{\max _{m}\left\{\left|\lambda_{i+/ 2}^{*, m}\right|\right\}}, \quad \gamma=\frac{\min \left\{\left|U_{s, i}\right|,\left|U_{s, i+1}\right|,\left|\delta U_{s, i+1 / 2}\right|\right\}}{\left|\delta U_{s, i+1 / 2}\right|}
$$

where $0 \leq \gamma \leq 1$. If the conserved variable is gradually varied, the coefficient $\gamma$ is 1 and the time step in (58) reduces to (54).

In the particular case $\gamma=0$ (58) predicts a null time step. Actually, this means that no flux can cross the associated edge $i+1 / 2$. When $\gamma=0$ it is necessary to modify the eigenvalues as follows

$$
\tilde{\lambda}_{i+1 / 2}^{m+, *}=\left\{\begin{array}{cc}
0 & \text { if } \quad \tilde{\lambda}_{i+1 / 2}^{m+, *}<0 \\
\tilde{\lambda}_{i+1 / 2}^{m+, *} & \text { otherwise }
\end{array} \quad \tilde{\lambda}_{i+1 / 2}^{m-, *}=\left\{\begin{array}{ccc}
0 & \text { if } \tilde{\lambda}_{i+1 / 2}^{m-, *}>0 \\
\tilde{\lambda}_{i+1 / 2}^{m-*} & \text { otherwise }
\end{array}\right.\right.
$$

When $\gamma<<1$ condition (58) force to strong restrictions in the magnitude of the time step and unacceptable computational costs. The reduction of the magnitude of the time step can be avoided by means of a conservative strategy based on the redistribution of updating fluxes, involving a the local time step $\Delta t_{U s, i}$, that replaces the $\gamma$ coefficient in (58). The local time step $\Delta t_{U s, i}$ is defined to keep the sign of the variable $U_{s, i}$. Expressing for each variable (50) as

$$
U_{s, i}^{n+1}=U_{s, i}^{n}+\Delta t\left(\Psi_{i, i-1 / 2}^{U s}+\Psi_{i, i+1 / 2}^{U s}\right), \quad \Psi_{i, i \pm 1 / 2}^{U s}=\frac{1}{\Delta x} \sum_{m=1}^{3}\left(\left(\widetilde{\lambda}^{\mp} \alpha-\beta^{\mp}\right) e_{1}\right)_{i, i \pm 1 / 2}^{m}
$$

In the case of require the positivity of the variable $U_{s, i} \Delta t_{U s, i}$ is computed as:

$$
\Delta t_{U s, i}=-\frac{U_{s, i}^{n}}{\Psi_{i, i-1 / 2, k}^{U s}+\Psi_{i, i+1 / 2, k}^{U s}}, \quad \Psi_{i, i \pm 1 / 2}^{U s}<0
$$

It is worth noting that the quantity defined in (61) is always positive due to the condition on the contributions in the denominator. In the case of require the positivity of more variables more $\Delta t_{U s, i}$ 's local steps are defined. Then a redistribution factor $r_{t, i}$ is computed as:

$$
r_{t, i}=\frac{\Delta t_{v}}{\Delta t_{\max }} \leq 1, \quad \Delta t_{v}=\min \left\{\Delta t_{\max }, \Delta t_{U s, i}\right\}
$$


where $\Delta t_{\max }$ is computed as in (79). When in the cell $i$ the ratio $r_{t, i}<1$, we propose to redefine the updating fluxes to cells at those edges where $\Psi_{i, i \pm 1 / 2}^{U s} \leq 0$ for one $U_{s}$ according to:

$$
\Psi_{i, i \pm 1 / 2}^{U s}=\omega_{U s 1}, \quad \Psi_{i \pm 1, i \pm 1 / 2}^{U s}=\Psi_{i \pm 1, i \pm 1 / 2}^{U s}+\omega_{U s 2}
$$

where, to preserve conservation, the quantities $\omega_{h 1}, \omega_{h 2}, \omega_{z 1}, \omega_{z 2}$ are

$$
\omega_{U s 1}=\Psi_{i, i \pm 1 / 2}^{U s} r_{t, i} \quad \omega_{U s 2}=\Psi_{i, i \pm 1 / 2}^{U s}\left(1-r_{t, i}\right)
$$

By means of this technique the variables are updating computing the time step as in the homogeneous case. In some cases negative values can be obtained, as the truly time step is various orders of magnitude smaller. In that case it is enough to set $\Delta t_{\max }=\frac{1}{2} \Delta t_{\max }$ and repeat the process until no negative values appear. This reduction has an irrelevant computational cost, generating always a solution.

\section{APPLICATION TO THE 1D SHALLOW WATER EQUATIONS}

\subsection{D mathematical model of the shallow water equations}

In this work the schemes are used to solve the following system of equations:

$$
\mathbf{U}=\left(h, q_{x}\right)^{T} \quad \mathbf{F}=\left(q, \frac{q^{2}}{h}+\frac{g h^{2}}{2}\right)^{T} \quad \mathbf{S}=\left(0,-g h \frac{\partial z}{\partial x}-\frac{\tau}{\rho_{w}}\right)^{T}
$$

where $h$ is the water depth, $g$ is the acceleration of the gravity, $q=u h$ the unit discharge with $u$ the averaged velocity. The bed shear-stress $\tau$ can be written using the expressions similar to the Chézy equation for open-channel hydraulics (Chow, 1973).

3.2. Application of the explicit upwind numerical scheme

As stated in $\S 2$, the mathematical properties of the hyperbolic system of equations include the existence of a Jacobian matrix. In the case of (139) it is convenient to work with the flux Jacobian matrix $\mathbf{J}$. Upwind schemes were first developed for the Euler equations (Toro, 1997). In those equations the numerical flux is first order homogeneous and (110) holds. This is not the case for the Saint-Venant equations (Vázquez-Cendón, 1999), and an approximate flux Jacobian matrix $\widetilde{J}$ has to be defined. The approximate matrix is

$$
\widetilde{\mathbf{J}}=\frac{\delta \mathbf{F}}{\delta \mathbf{W}}=\left(\begin{array}{cc}
0 & 1 \\
g \widetilde{h}-\widetilde{u}^{2} & 2 \widetilde{u}
\end{array}\right)
$$

The eigenvalues and eigenvectors of 60 are:

where

$$
\begin{array}{cc}
\tilde{\lambda}_{i+1 / 2}^{1}=(u+\widetilde{c})_{i, k} & \widetilde{\lambda}_{i+1 / 2}^{2}=(u-\widetilde{c})_{i, k} \\
\widetilde{\mathbf{e}}_{i, k}^{1}=\left(\begin{array}{c}
1 \\
\widetilde{u}+\widetilde{c}
\end{array}\right)_{i, k}, \quad \widetilde{\mathbf{e}}_{i, k}^{2}=\left(\begin{array}{c}
1 \\
\widetilde{u}-\widetilde{c}
\end{array}\right)_{i, k}
\end{array}
$$

$$
\widetilde{u}_{i+1 / 2}=\frac{u_{i} \sqrt{h_{i}+u_{i+1} \sqrt{h_{i+1}}}}{\sqrt{h_{i}+\sqrt{h_{i+1}}}}, \widetilde{c}_{i+1 / 2}=\sqrt{g \frac{h_{i}+h_{i+1}}{2}}
$$

The difference in vector $\mathbf{U}$ across the grid edge is and the expression of coefficients $\alpha_{i,+1 / 2}^{m}$ are:

$$
\alpha_{i+1 / 2}^{1,2}=\frac{\delta h_{i+1 / 2}}{2} \pm \frac{1}{2 \widetilde{c}_{i+1 / 2}}(\delta q-\widetilde{u} \delta h)_{i+1 / 2}
$$

The non conservative term $\mathbf{S}$ is included defining $\delta \mathbf{T}_{i+1 / 2}$, with 


$$
\delta \mathbf{T}_{i+1 / 2}=\left(\begin{array}{c}
0 \\
-g \widetilde{h} \delta z-\frac{\widetilde{\tau}}{\rho_{w}} \Delta x
\end{array}\right)_{i+1 / 2}
$$

Here it is remarkable that if the matrix $\mathbf{S}$ were constant the equality $\mathbf{S}(\mathbf{U})=\partial_{x} \mathbf{T}$ would be true. Obviously, this is not our case, but the linearization of the non conservative term can be considered locally for numerical approximation. Now, following (87) the coefficients $\beta^{m}$ are defined as:

$$
\beta^{1,2}=\mp \frac{1}{2}\left(\widetilde{c} \delta z+\frac{\widetilde{\tau} \Delta x}{\widetilde{c} \rho_{w}}\right)_{i+1 / 2}
$$

\subsection{Conservation properties and equilibrium at steady state}

The unified discretization of the source terms is successfully constructed when it ensures an exact balance in first order approximation (Bermudez and Vazquez-Cendon 1998). For that reason, the discretization of the fluxes and source terms proposed in $\S 3.1$ is here analysed. Steady state in the first order scheme is expressed as:

$$
\widetilde{\mathbf{J}}_{i+1 / 2} \delta \mathbf{U}_{i+1 / 2}=\delta \widetilde{\mathbf{T}}_{i+1 / 2}
$$

According to the form of the matrices involved, the first equation in (147) gives

$$
\delta(h u)_{i+1 / 2}=\delta(q)_{i+1 / 2}=0
$$

which means that the normal discharge is constant at the edge. The second line in (147) yields

$$
\delta h_{i+1 / 2}\left(\widetilde{c}^{2}-\widetilde{u}^{2}\right)_{i+1 / 2}+\delta q_{i+1 / 2}(2 \widetilde{u})_{i+1 / 2}=-(g \widetilde{h} \delta z)_{i+1 / 2}-\left(\frac{\widetilde{\tau} \Delta x}{\rho_{w}}\right)_{i+1 / 2}
$$

Considering (148) and expressing the friccion term in functions of the slope energy $S_{f_{i+1 / 2}}$,

$$
\left(\frac{\widetilde{\tau}}{\rho_{w}}\right)_{i+1 / 2}=\left(g \widetilde{h} S_{f}\right)_{i+1 / 2}
$$

the expression for the water depth profile for uniform flows appears:

$$
\delta h_{i+1 / 2}\left(1-F r_{i+1 / 2}^{2}\right)+\delta z_{i+1 / 2}=-\Delta x S_{i+1 / 2}
$$

where $F r_{i+1 / 2}$ is the Roe average Froude number in the normal edge direction, $F r_{i+1 / 2}=\widetilde{u}_{i+1 / 2} / \widetilde{c}_{i+1 / 2}$. Equation (152) can be derived directly enforcing

$$
\delta \mathbf{D}_{i+1 / 2}^{m}=0
$$

or $\left(\tilde{\lambda}^{-} \alpha-\beta^{-}\right)_{i+1 / 2}^{m}=0$ for $m=1$ and $m=2$. Therefore the discretization ensures equilibrium in steady state cases correctly for first order approximation, and the $\mathcal{G}$-Property is satisfied not only in the case of quiescence flow over discontinuous bed topography, also in the case of steady fluid in motion.

\subsection{Interpretation of the $\theta$ coefficient}

In the homogenous case, without source terms, $\theta_{i+1 / 2}^{ \pm}=1$, the original stability region is automatically recovered. The case $\theta_{i+1 / 2}^{ \pm}=0$, correspond to the equilibrium case ( $\mathcal{G}$-Property is satisfied) and the numerical scheme becomes unconditionally stable. On the other hand, when $\theta_{i+1 / 2}^{ \pm}<0$ the positivity requirements over the variables are satisfied if the stability region is defined using:

$$
\gamma=\frac{\min \left\{h_{i}, h_{i+1},\left|\delta h_{i+1 / 2}\right|\right\}}{\left|\delta h_{i+1 / 2}\right|}, \quad \theta_{i+1 / 2}^{ \pm}<0
$$


Condition (78) is of special relevance when managing wetting and drying fronts. In the special case, $h_{i+1}=0$ or $h_{i}=0, \gamma$ is nil and it is necessary to modify the eigenvalues as in (59) otherwise positivity condition over the mixture depth is not ensured. As no flux can cross the associated edge $i+1 / 2$ the following condition must be applied to the future solution at the cells sharing edge $i+1 / 2$ :

$$
u_{i}^{n+1}=u_{i+1}^{n+1}=0
$$

To avoid strong restrictions in the magnitude of the time step when $\gamma<<1$ the redistribution factor is computed as:

$$
r_{t, i}=\frac{\Delta t_{v}}{\Delta t_{\max }} \leq 1, \quad \Delta t_{v}=\min \left\{\Delta t_{\max }, \Delta t_{h, i}\right\}
$$

where $\Delta t_{h, i}$ is computed following (61).

Also, to preserve conservation it is necessary to impose (93) in the edges where $\Psi_{i \pm 1 / 2}^{h} \leq 0$ and $r_{t, i} \leq 1$.The redefinition of the fluxes according to (63) is done in the cell edges where $\Psi_{i, i \pm 1 / 2}^{h} \leq 0$.

Near wetting/drying fronts, characterized by small values of water depth, the bed friction term may dominate over any other term, leading to numerical instabilities. When $0<\gamma<1$ numerical instabilities are avoided by requiring that friction alone is not able to change the sign of the discharge, so the following conditions are enforced over the unit discharge function $h u$

$$
(h u)_{i}^{n+1} \geq 0 \quad(h u)_{i}^{n},(h u)_{i \pm 1}^{n} \geq 0 \quad \text { or } \quad(h u)_{i}^{n+1} \leq 0 \quad(h u)_{i}^{n},(h u)_{i \pm 1}^{n} \leq 0
$$

These conditions must be included to determine the maximum allowable time step, otherwise the numerical scheme leads to numerical instabilities. Let us assume without lost of generality the one-dimensional case, where the updated value can be expressed as:

that can be rewritten as

$$
(h u)_{i}^{n+1}=(h u)_{i}^{n}-\left(g \widetilde{h} S_{f} \Delta x\right)_{k}^{n} \frac{\Delta t}{\Delta x}
$$

$$
(h u)_{i}^{n+1}=(h u)_{i}^{n}\left[1-\frac{\left(g \widetilde{h} S_{f}\right)_{k}^{n}}{(h u)_{i}^{n}} \Delta t\right]
$$

The second term on right hand of (91) must be positive to ensure (89). Hence, in general, the time step $\Delta t_{k}$, taking into account also condition (86), is limited by

$$
\Delta t_{i+1 / 2}=\min \left\{\left|\frac{\widetilde{u}_{i+1 / 2}}{g S_{f, i+1 / 2}}\right|, \frac{\Delta x}{\max _{m}\left\{\left|\widetilde{\lambda}_{i+1 / 2}^{m}\right|\right\}}\right\}_{i+1 / 2}
$$

to prevent instabilities.

\section{APPLICATION TO THE 1D SHALLOW WATER EQUATIONS WITH SOLUTE TRANSPORT}

3.1. 2D mathematical model of the shallow water equations with solute transport

In this work the schemes are used to solve the following system of equations:

$$
\mathbf{U}=\left(h, q_{x}, h \phi\right)^{T}, \quad \mathbf{F}=\left(q, \frac{q^{2}}{h}+\frac{g h^{2}}{2}, q \phi\right)^{T} \quad \mathbf{S}=\left(0,-g h \frac{\partial z}{\partial x}-\frac{\tau}{\rho_{w}}, 0\right)^{T}
$$

where $\phi$ is the depth averaged solute concentration. 


\subsection{Application of the explicit upwind numerical scheme}

As stated in $\S 2$, the mathematical properties of the hyperbolic system of equations include the existence of a Jacobian matrix. The eigenvalues and eigenvectors are:

$$
\begin{aligned}
& \widetilde{\lambda}_{i+1 / 2}^{1}=(\widetilde{u}+\widetilde{c})_{i+1 / 2} \quad \widetilde{\lambda}_{i+1 / 2}^{2}=(\widetilde{u}-\widetilde{c})_{i+1 / 2} \quad \widetilde{\lambda}_{i+1 / 2}^{3}=\widetilde{u}_{i+1 / 2} \\
& \widetilde{\mathbf{e}}_{i, k}^{1}=\left(\begin{array}{c}
1 \\
\tilde{u}+\widetilde{c}
\end{array}\right)_{i, k}, \quad \widetilde{\mathbf{e}}_{i, k}^{2}=\left(\begin{array}{c}
1 \\
\tilde{u}-\widetilde{c}
\end{array}\right)_{i, k} \quad \widetilde{\mathbf{e}}_{i, k}^{3}=\left(\begin{array}{l}
0 \\
1
\end{array}\right)_{i, k}
\end{aligned}
$$

where

$$
\widetilde{\phi}_{i+1 / 2}=\frac{\widetilde{\phi}_{i} \sqrt{h_{i}+\widetilde{\phi}_{i+1} \sqrt{h_{i+1}}}}{\sqrt{h_{i}}+\sqrt{h_{i+1}}}
$$

The difference in vector $\mathbf{U}$ across the grid edge is and the expression of coefficients $\alpha_{i,+1 / 2}^{m}$ are:

$$
\alpha_{i+1 / 2}^{1,2}=\frac{\delta h_{i+1 / 2}}{2} \pm \frac{1}{2 \widetilde{c}_{i+1 / 2}}(\delta q-\tilde{u} \delta h)_{i+1 / 2} \quad \alpha_{i+1 / 2}^{3}=\delta(h \phi)_{i+1 / 2}-\widetilde{\phi}_{i+1 / 2}(\delta h)_{i+1 / 2}
$$

Now, following (87) the coefficients $\beta^{m}$ are defined as:

$$
\beta^{1,2}=\mp \frac{1}{2}\left(\widetilde{c} \delta z+\frac{\widetilde{\tau} \Delta x}{\widetilde{c} \rho_{w}}\right)_{i+1 / 2}, \quad \beta^{3}=0
$$

\subsection{Interpretation of the $\theta$ coefficient}

When moving to the coupled set of water flow and solute transport equations, $\gamma$ is defined with the same purpose in this case as:

$$
\begin{gathered}
\gamma=\min \left\{\gamma_{h}, \gamma_{h \phi}\right\} \\
\gamma_{h}=\frac{\min \left\{h_{i}, h_{i+1},\left|\delta h_{i+1 / 2}\right|\right\}}{\left|\delta h_{i+1 / 2}\right|}, \quad \gamma_{h \phi}=\frac{\min \left\{(h \phi)_{i},(h \phi)_{i+1},\left|\delta(h \phi)_{i+1 / 2}\right|\right\}}{\left|\delta(h \phi)_{i+1 / 2}\right|} \quad \theta_{i+1 / 2}^{ \pm}<0
\end{gathered}
$$

In presence of solute fronts, that is clean/mixed water boundaries with continuous water level surface this leads to unrealistic results in the solute advance. If this fact is not considered, negative values of solute mass and concentration can be obtained and the necessity to tune and alter the scheme results becomes necessary. This can be avoided using again a conservative redistribution of the updating contributions (Murillo et al. 2006a) that ensures adequate bounding properties over the solute concentration.

Now the redistribution factor is computed as:

$$
r_{t, i}=\frac{\Delta t_{v}}{\Delta t_{\max }} \leq 1, \quad \Delta t_{v}=\min \left\{\Delta t_{\max }, \Delta t_{h, i}, \Delta t_{h \phi, i}\right\}
$$

and we propose to redefine the updating fluxes to cells at those edges where $\Psi_{i, i \pm 1 / 2}^{h} \leq 0$ according to (63) over the water depth and over the solute mass.

\section{APPLICATION TO THE 1D BED LOAD SEDIMENT TRANSPORT}

The mathematical model used in this work is based in the differential form of the equations described by Hudson involving the friction term. In this model the solid phase has a velocity equal to the liquid one and presents a uniform distribution over the flow depth. No exchanges through the bottom interface are considered. The system of equations are represented by

$$
\mathbf{U}=\left(\begin{array}{c}
h \\
u h \\
z
\end{array}\right), \mathbf{F}=\left(\begin{array}{c}
h u \\
\left(u^{2} h+\frac{1}{2} g h^{2}\right) \\
\xi q_{l}
\end{array}\right), \mathbf{S}=\left(\begin{array}{c}
0 \\
-g h \frac{\partial z}{\partial x}-\frac{\tau}{\rho_{w}} \\
0
\end{array}\right)
$$


Where $h$ is the water depth, $u$ is the depth averaged velocity, $\mathrm{z}$ is the bottom elevation, $\xi=1 /(1-\varepsilon)$ where $\varepsilon$ is the porosity, g is gravity, $\rho_{w}$ is the density of the water, $\tau$ is the bed shear-stress, and $q_{l}$ is the total volumetric sediment rate. The sediment transport flux is defined using the Grass formulation (Grass,2000)

$$
q_{l}=A u\left(u^{2}+v^{2}\right)^{1 / 2(m-1)}
$$

where $A$ is dimensional constant usually determined by experimental data and $1 \leq m \leq 4(3,4)$. In this work $m$ is set constant and equal to 3. It is remarkable that to avoid the singularity in the Jacobian matrix of the flux, the flux $\mathbf{F}$ and the slope term $\mathbf{S}_{1}$ are modified as follows (Hudson, 2000)

$$
\mathbf{F}=\left(\begin{array}{c}
h u \\
u^{2} h+\frac{1}{2} g h^{2}+g h z \\
q_{l}
\end{array}\right), \mathbf{S}=\left(\begin{array}{c}
0 \\
g z \frac{\partial h}{\partial x}-\frac{\tau}{\rho_{w}} \\
0
\end{array}\right)
$$

leading to the following Jacobian matrix

$$
\mathbf{J}=\frac{\partial \mathbf{F}}{\partial \mathbf{W}}=\left(\begin{array}{ccc}
0 & 1 & 0 \\
g(h+z)-u^{2} & 2 u & g h \\
-u d & d & 0
\end{array}\right)
$$

with $d_{1}=3 A \xi u^{2} h^{-1}$. Hudson defined an approximate Jacobian matrix where the averaged eigenvalues where computed solving the following third order polynomial,

$$
P(\widetilde{\lambda})=\widetilde{\lambda}^{3}-2 \widetilde{u} \widetilde{\lambda}^{2}+\widetilde{u}^{2}-[g(\widetilde{h}+\widetilde{z}+\widetilde{h} \widetilde{d})] \widetilde{\lambda}+g \widetilde{h} \widetilde{u} \widetilde{d}=0
$$

where

$$
\widetilde{d}_{i+1 / 2}=c_{b} \beta \frac{\sqrt{h_{i+1}}+\sqrt{h_{i}}}{\sqrt{h_{i+1} h_{i}}+\sqrt{h_{i} h_{i+1}}}\left(u_{i+1}^{2}+u_{i}^{2}+u_{i} u_{i+1}\right)
$$

leading to three real and distinct eigenvalues. Therefore the problem is strictly hyperbolic. Two eigenvalues have the same sign of the particle velocity $\widetilde{u}$, one is opposite. We name it in ascending order: with $\tilde{u}>0, \tilde{\lambda}^{1}<0, \tilde{\lambda}^{2} \geq 0, \tilde{\lambda}^{3}>0$ and $\widetilde{\lambda}^{3}>\tilde{\lambda}^{2}$, while $\tilde{\lambda}^{2}=0$ only if $\tilde{u}=0$, the condition of fluid at rest. The right eigenvector associated to each $\lambda^{m}$ is

$$
\widetilde{\mathbf{e}}_{i+1 / 2}^{m}=\left(\begin{array}{c}
1 \\
\widetilde{\lambda}^{m} \\
\left(-g(\widetilde{h}+\widetilde{z})+\left(\widetilde{\lambda}^{m}-\widetilde{u}\right)^{2}\right)(g \widetilde{h})^{-1}
\end{array}\right)_{i+1 / 2} m=1,2,3
$$

where the coefficient $\alpha_{i+1 / 2}^{m}$ is equal to

$$
\alpha_{i+1 / 2}^{m}=\left[\frac{\left(\widetilde{\lambda}^{a} \widetilde{\lambda}^{b}+g(\widetilde{h}+\widetilde{z})-\widetilde{u}^{2}\right) \delta h+\left(2 \widetilde{u}-\widetilde{\lambda}^{a}-\widetilde{\lambda}^{b}\right) \delta(h u)+g \widetilde{h} \delta z}{\left(\widetilde{\lambda}^{k}-\widetilde{\lambda}^{a}\right)\left(\widetilde{\lambda}^{k}-\widetilde{\lambda}^{b}\right)}\right]_{i+1 / 2} \quad m=1,2,3
$$

with $a \neq b \neq k$. The different $\beta^{m}$ coefficients are computed as

$$
\beta_{i+1 / 2}^{m}=\left(\frac{\left(2 \widetilde{u}-\widetilde{\lambda}^{a}-\widetilde{\lambda}^{b}\right)\left(g z \delta h-\widetilde{\tau} \Delta x / \rho_{w}\right)}{\left(\widetilde{\lambda}^{m}-\widetilde{\lambda}^{a}\right)\left(\widetilde{\lambda}^{m}-\widetilde{\lambda}^{b}\right)}\right)_{i+1 / 2} \quad m=1,2,3
$$

In the case that $\widetilde{u}_{i+1 / 2}$ becomes nil, $u_{i+1}=u_{i}=0, \widetilde{\lambda}^{2}$ becomes nil and $\widetilde{\mathbf{e}}^{2}=\mathbf{0}$, so the linearization in (30) fails. Also in the case that $h_{i+1}$ or $h_{i}$ are nil the averaged quatities in (24) become useless. In such cases we consider that no sediment transport is performed and the system of equations becomes the shallow water equations. 
The positivity requirements over the water depth is satisfied defining $\gamma$ as in (78). To avoid strong restrictions in the magnitude of the time step when $\gamma<<1$, the redistribution factor is computed as in (88). The redefinition of the fluxes according to (63) is done in the cell edges where $\Psi_{i, i \pm 1 / 2}^{h} \leq 0$ over the water depth and bottom elevation. Also the same restrictions imposed by the friction term must be observed.

\section{APLICATIONS}

\subsection{Long wave resonance in a parabolic basin.}

The analytical solution of a long wave, driven by gravity and resonating in a frictionless, dry circular parabolic basin was presented by Thacker (Thacker, 1981) for the shallow water equations, where the free surface displacement is given by

$$
\zeta(r, t)=\zeta_{o}\left(\left(1-A^{2}\right)^{1 / 2} B^{-1}-1-r^{2} a^{-2}\left\{\left(1-A^{2}\right) B^{-2}-1\right\}\right)
$$

and the bottom elevation is given as

$$
z(r, t)=-\zeta_{0}\left(1-r^{2} a^{-2}\right)
$$

where $A=\left(a^{4}-r_{o}^{4}\right)\left(a^{4}+r_{o}^{4}\right)^{-1}, B=1-A \cos \omega t, \omega=a^{-1} \sqrt{8 g} \zeta_{o}, \zeta_{o}$ is the center point water depth, $r$ is the distance from the center point, $a$ is the radial distance from the center point to the zero elevation on the shoreline and $r_{o}$ is the distance from the center point to the point where the water depth is initially nil. Those values are represented in Fig. 2. The domain shape of the two previous examples is used again and the numerical values are $\zeta_{o}=20.0 \mathrm{~m}$, $r_{o}=1200 \mathrm{~m}, a=1500 \mathrm{~m}$. The domain is divided in using cells with $\Delta x=25 \mathrm{~m}$.

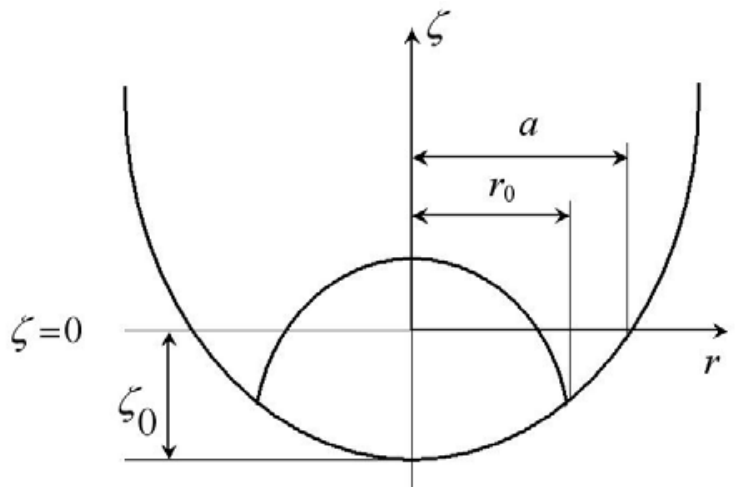

Figure 2. Initial free surface and water depth profile for the parabolic basin test.

The water surface movement will be simulated together with an initial solute concentration:

$$
\phi\left(r, t_{o}\right)=\phi_{o} \exp \left(-r\left(2 r_{o}\right)^{-1}\right)
$$

There is no analytical solution for the solute concentration evolution in time but, if no diffusion is assumed, the solution for each $T$ oscillation period will be:

$$
\phi(r, t=T K)=\phi_{o} \exp \left(-r\left(2 r_{o}\right)^{-1}\right), \quad K=1, \ldots, \infty
$$

The numerical experiment is performed using $\phi_{o}=1$. This test case illustrates the concepts described in this work, as it includes wetting/drying fronts and the generation of dry regions from wet areas. In particular, the advance of the wetting/drying front is produced in the first half period, during the wave expansion, while during the wave contraction both wetting/drying fronts and drying process are present.

Figure 3 shows the water depths given by the exact solution and by the proposed method at times, $1 / 4 T, 2 / 4 T, 3 / 4 T, T, 3 / 2 T, 2 T, 5 / 2 T, 3 T, 7 T / 2$ and $4 T$.. The simulated results prove in 
every case in good agreement with the analytical solution, including water depth and inundated area, even in the fourth oscillation. Figure 4 shows the comparison between the exact and numerical solution for the solute concentration distribution at times $T, 2 T, 3 T$ and $4 T$. Better accuracy in the final solution can be achieved reducing the size of the cells generating a better representation of the bottom elevation.
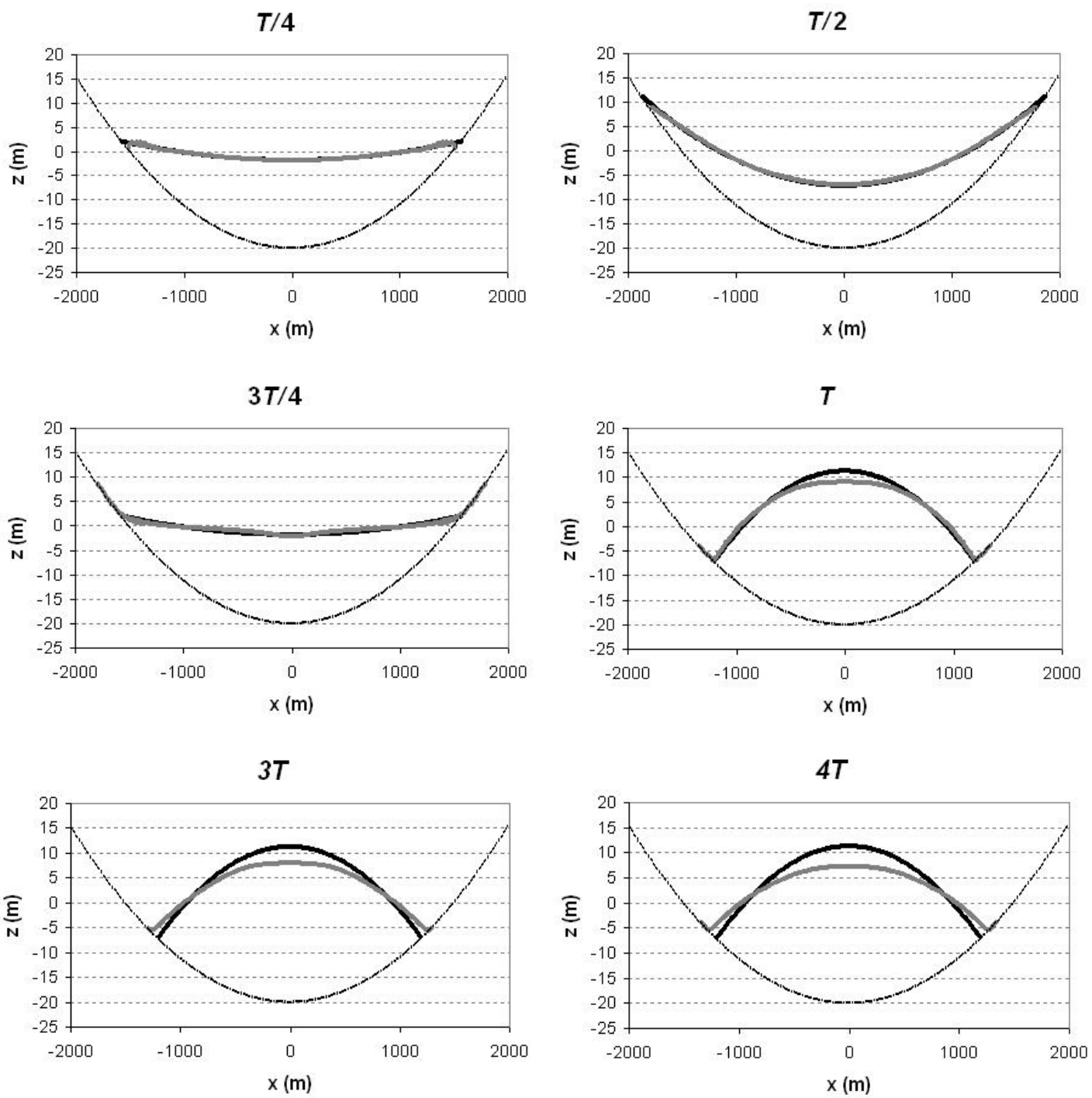

-exact - computed

Figure 3. Water elevation surface (in meters): exact and simulated for times 1/4T, 2/4T, 3/4T, $T, 3 T$ and $4 T$. 

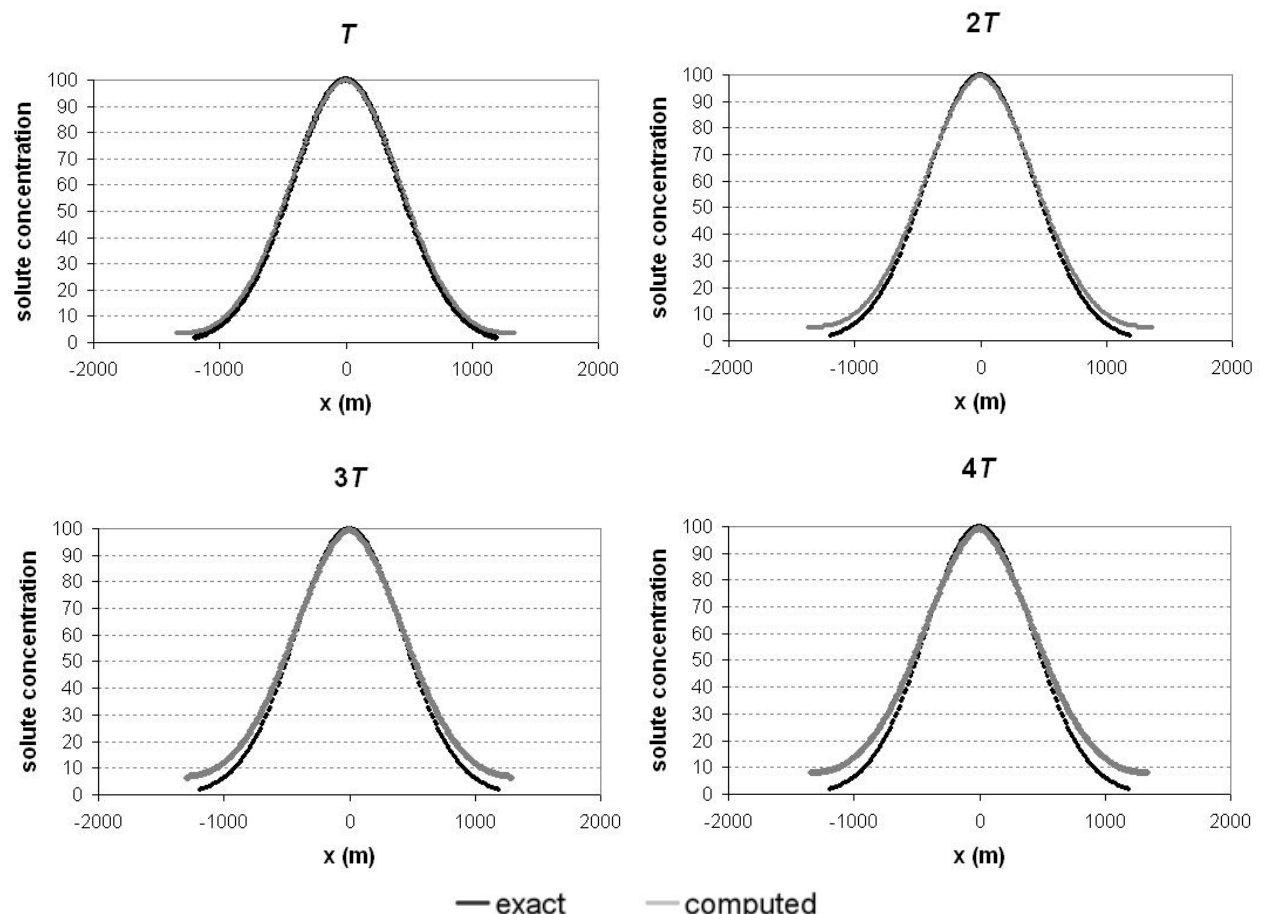

Fig. 4. Solute distribution results and exact solution at times $T, 2 T, 3 T$ and $4 T$.

\subsection{Equilibrium.}

In the following test case the bed evolution to equilibrium of a channel departing from a situation of still water is analysed. A domain 100 meters long is divided in cells with $\Delta x=1 \mathrm{~m}$, imposing $u=0, z=0 \mathrm{~m}$ and $h=2$. From the beginning of the simulation a constant discharge is imposed upstream, $h u=10 \mathrm{~m}^{2} / \mathrm{s}$, while downstream a constant water level surface $h+z=2$ $\mathrm{m}$ is imposed, assuming $\xi=0.5$ and $A=0.05$. The shear stress is defined using the Manning equation:

$$
\tau=\rho_{w} g n^{2} u|u| h^{-1 / 3}
$$

with $\mathrm{n}=0.04$. Figure 5 shows the final bed and water level surface equilibrium slopes. In case of use a pointwise discretization for the friction term any remarkable differences appear in the bed and water surface elevations, but for the pointwise simulation discharge varies along the domain, as Figure 6 shows. 


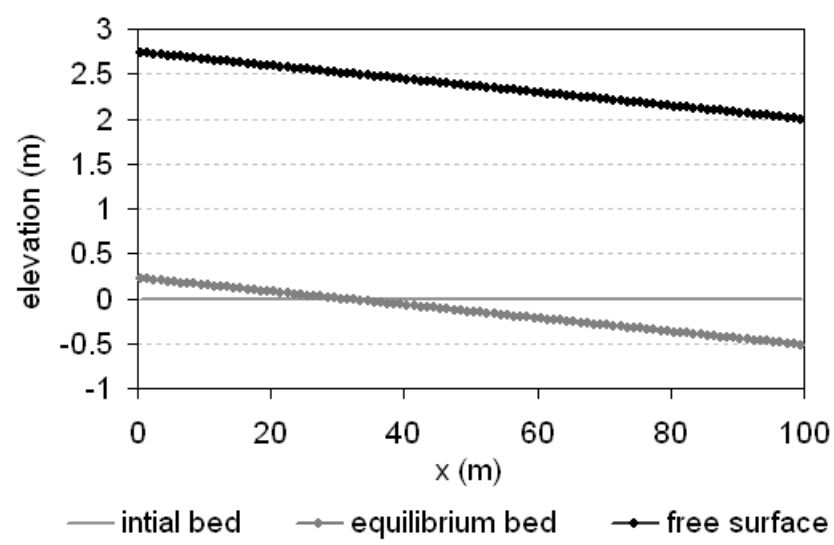

Figure 5. Final bed and water surface levels in equilibrium.

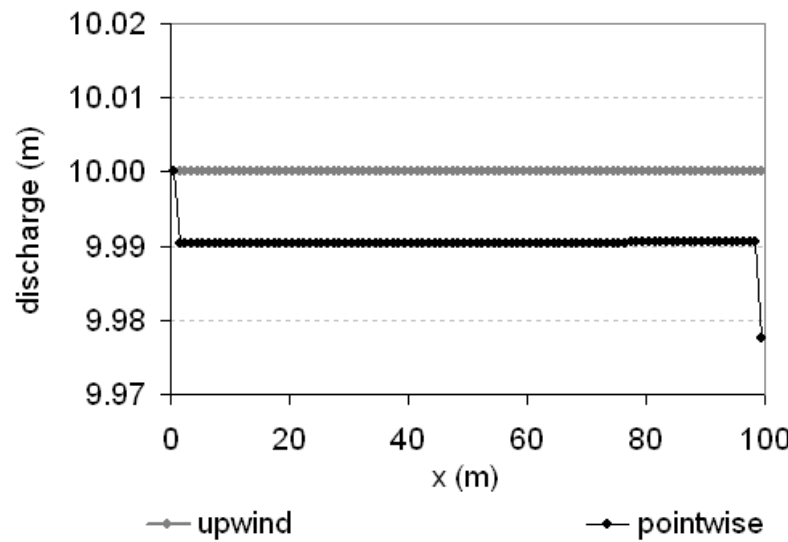

Figure 6. Equilibrium discharge using an upwind and a pointwise formulation foe the friction term.

\subsection{Dam break over movable bed.}

The model proposed by Hudson is applied to erosional dam-break problems. A body of water at rest of constant depth $h_{o}$, is realised over a flat erodible bed channel extending on both sides. To different experiments are used to check the performance of the numerical scheme. The first was performed in the Université Catholique de Louvain (Louvain-la-Neuve) and the second in the National Taiwan University (Taipei) with coarse granular material of different densities (Capart and Fraccarollo, 2002). In the Louvain experiment an intermediate density for the material was chosen $\left(s=\rho_{s} / \rho_{w}=1.540\right)$ while in the Taipei experiment a much lighter material was choose $(s=1.048)$, in both cases lighter than natural sediment $(s \approx 2.65)$. The initial water depth in both cases is $h_{o}=0.01 \mathrm{~m}$. It is possible to define the sediment load as (Rosatti and Fraccarollo, 2006, Summer et al., 1996) we define

$$
q_{l}=c_{b} \beta u^{3}
$$

where $c_{b}$ is volumetric concentration of sediments in the bed $\left(c_{b}=1-\varepsilon\right)$. In both cases $c_{b}=0.5$, and the shear stress is obtained using an expression similar to the Chézy equation:

$$
\tau=\rho_{w} s C_{f} u|u|
$$

Following Fraccarollo and Capart2002 $\beta$ is defined as

$$
\beta_{s}=s C_{f}\left(g c_{b}(1-s) \tan \varphi\right)^{-1}
$$


using in both cases $C_{f}=0.014, \tan \varphi=0.5$. In the Louvain case $\beta$ is 0.016 and in the Taipei case 0.125 . With this formulation it is possible to estimate the depth of the rich sediment layer in movement,

$$
h_{s}=\beta_{s} u^{2}
$$

Figure 7 compares the experimental data and the computational solution for the Louvain test case, at times $t=5 t_{o}, 7.5 t_{o}, 10 t_{o}$, being $t_{o}$ the geomorphologic time scales, approximately $0.101 \mathrm{~s}$. The main differences are attributed to the fact that in the mathematical model mass and momentum exchanges through the bottom interface are considered.

In the Taipei test case the exchanges through the bottom interface can not be neglected, and the model predicts unrealistic solutions as Figure 8 shows for time $t=3 t_{\text {o }}$.
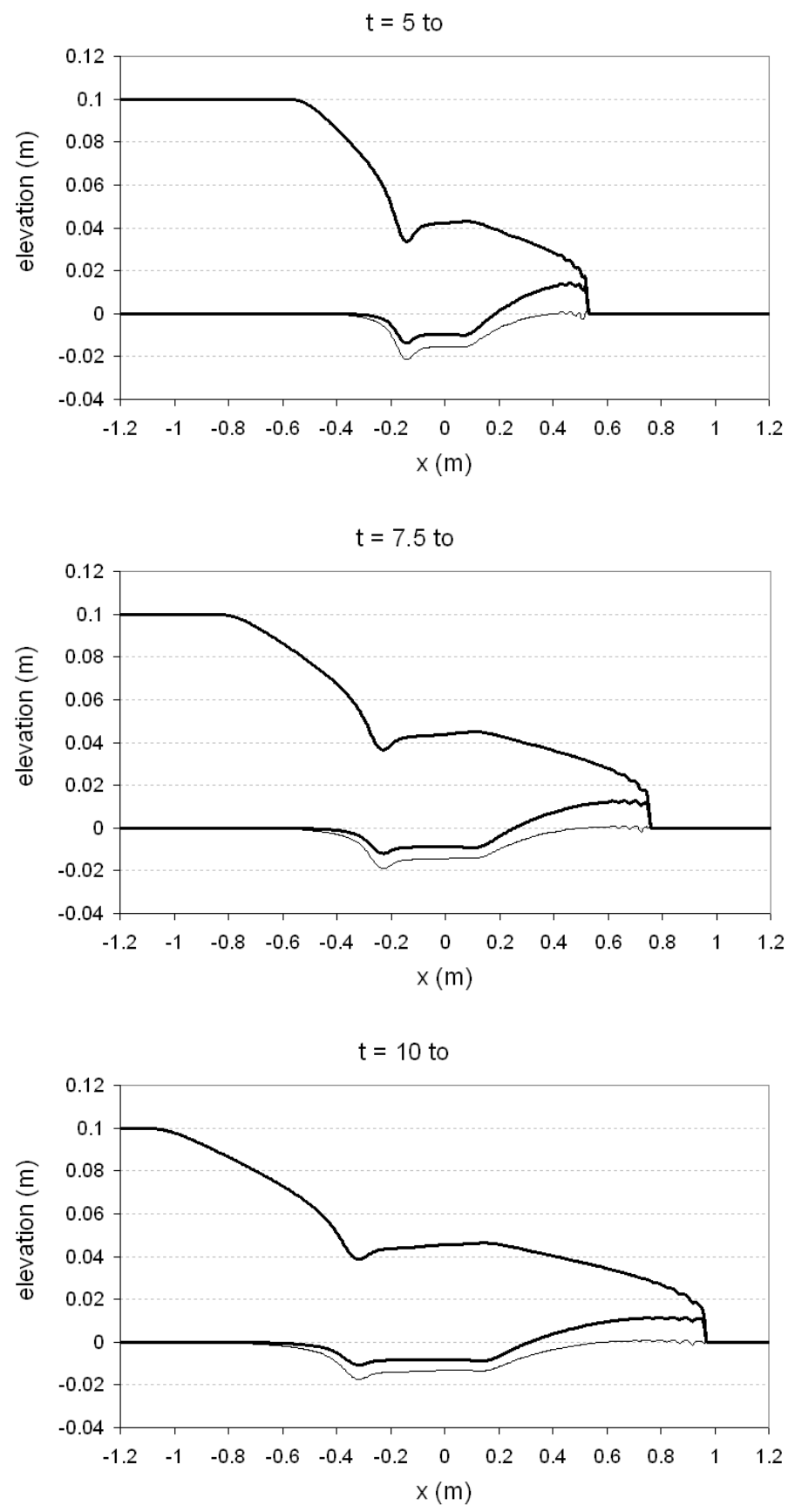
Figure 7. Experimental data and the computational solution for the Louvain test case, at times $t=5 t_{o}, 7.5 t_{o}, 10 t_{o}$.

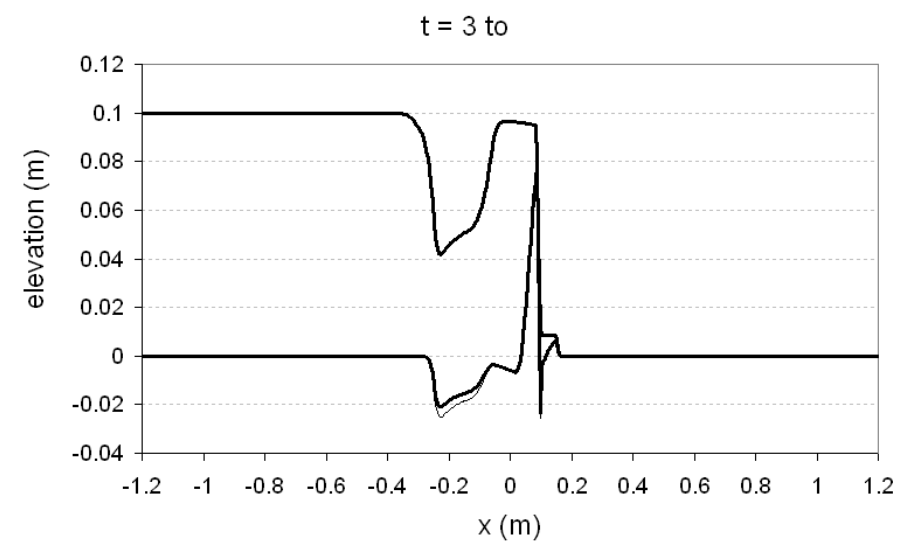

Figure 8. Experimental data and the computational solution for the Louvain test case, at time $t$

$$
=3 t_{o} \text {. }
$$

\section{CONCLUSIONS}

\section{REFERENCES}

L. Fraccarollo, Capart H., Riemann wave description of erosional dam-break flows, J. Fluid Mech., 372(1998), 165-187.

B.M.Summer, A.Kozakievicz, J. Fredsoe, R. Deigaard, Velocity and concentration profiles in sheet-flow layer of movable bed. Journal of Hydraulic Eng. 122 (1996) 549-558

Hudson J. Numerical techniques for morphodynamic modelling. Ph.D. Thesis, University of reading, October 2002.

N. Črnjarič-Ž̌ic, S. Vukovié and L. Sopta N. Extension of ENO and WENO schemes to onedimensional transport equations. Comut. Fluids 33(2004) 31-56.

G.Rosatti, L. Fraccarollo.A well-balanced approach for flow over movibile-bed with high sediment transport. Journal of Computational Physics, 220, 312-338 (2006).

J. Hudson and P.K. Sweby, Formulations for Numerically Approximating Hyperbolic Systems Governing Sediment Transport. Journal of Scientific Computing, 19, 225-252, (2003)

J. Hudson , P.K. Sweby. A high-resolution scheme for the equations governing 2D bed-load sediment transport, International Journal for Numerical Methods in Fluids 47, 1085 - 1091, (2005) 